Research Article

\title{
¿Desaparecidas o escondidas? Análisis de la presencia de las ciencias sociales y las humanidades en las versiones online de The Guardian, El País y Público
}

\section{Disappeared or hidden? Analysing the presence of social sciences and humanities in online versions of The Guardian, El País and Público}

\author{
Maider Eizmendi Iraola ${ }^{1 *}$ and Simón Peña-Fernandez ${ }^{1}$ \\ 1Universidad del País Vasco, Facultad de Ciencias Sociales y de la Comunicación, Barrio Sarriena, 48940 \\ Leioa, España. \\ *Correspondencia: maider.eizmendi@ehu.eus
}

\begin{abstract}
Resumen: La histórica división y jerarquización de los distintos saberes se ha trasladado también al periodismo científico, que ha primado habitualmente ciertas áreas como la ciencia, la tecnología, la ingeniería o las matemáticas (CTIM). En este contexto, el objetivo de este estudio ha consistido en analizar la presencia de las ciencias sociales y las humanidades en los medios online, partiendo de la idea de que, dadas sus características, los cibermedios son herramientas idóneas para el periodismo científico y se prestan a incluir nuevos temas. Mediante el análisis de contenido, se han estudiado 208 informaciones sobre ciencia publicadas en El País (España), Público (Portugal) y The Guardian (Reino Unido). Los resultados indican que la presencia de las ciencias sociales y las humanidades es menor que la de las ciencias duras y asciende a una de cada tres informaciones analizadas, siendo la historia y la economía los temas más presentes. Los datos también indican que las noticias sobre las ciencias sociales y las humanidades tienden a utilizarse en mayor medida como fuentes de autoridad, con una presencia más dispersa en diferentes secciones del medio, frente a un mayor valor noticioso de los resultados de las investigaciones en las áreas de la ciencia, la tecnología, la ingeniería o las matemáticas, que tienden a aparecer con mayor frecuencia en la sección específica de cada medio. Por todo ello, el protagonismo de las ciencias sociales y las humanidades tiende a quedar diluido en las páginas de los medios de comunicación frente al resto de las disciplinas científicas.
\end{abstract}

Palabras clave: medios online; periodismo científico; ciencias sociales; ciencias duras.

Abstract: The traditional division and hierarchy of the different areas of knowledge in the field of science has also been transferred to the study of science journalism, which has given priority to certain areas, and has shown a superior interest in science, technology, engineering and mathematics (STEM). In this context, the objective of this study has been to analyze the presence of social sciences and humanities in online media, based on the idea that, given their characteristics, they are ideal tools for scientific journalism, since they allow the inclusion of new topics. The results indicate that the presence of social sciences and humanities is less than that of hard sciences and increases one of every three pieces of information analyzed 
being history and economics as the most present topics. The data also indicate that the news on the social sciences and the humanities is to be used to a greater extent as sources of authority, with a more dispersed presence in different sections. On the contrary, the results of investigations in the areas of science, technology, engineering or mathematics have greater news value, and appear with greater frequency in the specific section of each media. Throughout, the prominence of social sciences and the humanities tends to be diluted in the pages of the media in relation to the rest of the scientific disciplines.

Keywords: online media; science journalism; social science; hard science.

\section{Introducción}

La red es una herramienta idónea para el periodismo científico (Brossard, 2013), cuya máxima prioridad desde su génesis ha sido acercar el conocimiento a la sociedad (Fernández de Lis, 2014). Las características intrínsecas de los cibermedios -la multimedialidad, la hipertextualidad y la interactividad (Deuze, 2001; Salaverría, 2005)- junto con lo que otros autores han denominado memoria o documentación (Daltoé, 2003) permiten profundizar en las principales singularidades atribuidas al periodismo científico: la necesidad de ofrecer los hechos noticiosos en su contexto (Alcíbar, 2004) y la importancia de las fuentes informativas (Elías, 2008). Asimismo, los medios digitales representan una oportunidad para experimentar con una nueva forma de comunicación y para abrir el espacio a nuevos temas (Franquet et al., 2007).

Estas potencialidades, sin embargo, están obligadas a lidiar con las dificultades y transformaciones que experimenta el periodismo en general y el periodismo científico en particular (Peters et al., 2014). El modelo de negocio de las empresas comunicativas está en crisis y este hecho repercute tanto en la situación laboral de los profesionales de la comunicación científica (Bauer et al., 2013; Schäfer, 2017), como en los contenidos que elaboran (Russell, 2010).

$\mathrm{Si}$ bien es cierto que la ciencia en general nunca ha sido una prioridad para los medios de comunicación (Dunwoody, 2008), la crisis financiera y la que, específicamente, acusan los medios de comunicación, ha provocado la desaparición de suplementos y secciones específicas sobre este ámbito y ha reducido la plantilla de los que han conseguido mantenerse (Schafër, 2017; Semir, 2010). Por ello, a pesar de que se investiga más que nunca, la cobertura que los medios han venido ofreciendo es menor (Cortiñas \& Alonso, 2014), por lo que los y las periodistas tienen un reto mayúsculo para transmitir los avances, contextualizándolos y explicando su magnitud (LópezCantos et al., 2020). Ciertos estudios, sin embargo, apuntan a que esta tendencia se está invirtiendo (Olvera-Lobo \& López-Pérez, 2015), aunque todavía está por ver la repercusión que puede tener la crisis derivada del Covid-19 en la relación entre los medios de comunicación y la ciencia, teniendo en cuenta que la pandemia ha tenido un efecto "disruptivo" sobre el periodismo (Casero-Ripollés, 2020: 22).

A los problemas citados hay que añadirle un descenso en la credibilidad y la calidad de la información científica (Bauer et al., 2013), provocado fundamentalmente porque los nuevos medios exigen rapidez y ello dificulta la elaboración de contenidos originales, la contextualización de los hechos y la verificación de las fuentes (Williams \& Clifford, 2009). La diversificación y el aumento de emisores de contenidos científicos (Peters et al., 2014; Trench, 2007) ha provocado, además, que la competencia entre diferentes medios sea mayor y se opte por contenidos vinculados al entretenimiento (Francescutti, 2018) y por un tratamiento sensacionalista de los hechos (De Semir, 2015; Molek-Kozakowska, 2017), que busca la aceptación por parte de un número mayor de consumidores. No obstante, otros autores inciden en que precisamente la crisis y el aumento de la competencia ha provocado un incremento de los contenidos analíticos, que inciden en el contexto y la comprensión por parte de los consumidores (Dunwoody, 2020: 473).

El ciberperiodismo es una de las áreas más fructíferas dentro de los estudios sobre comunicación (Salaverría, 2005) y, por supuesto, también en el ámbito del periodismo científico, en el que, tal y como se ha citado, ofrece multitud de oportunidades. Sin embargo, las 
investigaciones sobre esta área en los cibermedios no son muy numerosas (Brossard \& Schefeule, 2013; Olvera-Lobo \& López-Pérez, 2015) y, por lo tanto, se presta a un análisis más exhaustivo.

Estudios realizados acerca de la cobertura de la ciencia han demostrado que la información científica es homogénea (Granado, 2011) y selectiva (Olvera-Lobo \& López-Pérez, 2015), ya que su tratamiento no difiere entre distintos medios y se centra en temáticas concretas. La física es una de las ramas más representadas junto con la medicina (Meneses, 2016; Segado et al., 2018; Spina \& Díez, 2019). Precisamente, varios trabajos destacan el manifiesto interés de los medios por las ciencias naturales $y$, fundamentalmente, por los temas de salud, de ahí que autores como Bucchi y Mazzolini (2003) se hayan referido a la "medicalización" de las noticias científicas.

A este respecto, la mayoría de los estudios han centrado su atención exclusivamente en el tratamiento de las ciencias naturales y en las áreas denominadas CTIM (Ciencia, Tecnología, Ingeniería y Matemáticas), también conocidas como STEM por su acrónimo en inglés (Science, Technology, Engineering and Mathematics), descartando el análisis de las ciencias sociales y las humanidades. De hecho, a la hora de ofrecer una definición sobre el periodismo científico, autores como Elías (2008) limitan a las ciencias naturales las temáticas que han de abordar los profesionales del periodismo científico.

Por lo tanto, uno de los primeros cometidos a la hora de plantear un análisis acerca del mensaje periodístico sobre la ciencia es limitar la propia definición del objeto a estudiar. El debate sobre esta cuestión está directamente relacionado con la división de las distintas áreas del conocimiento y el estatus del que goza cada una de ellas. Históricamente, se han separado los distintos saberes y las disciplinas y, no solo eso, también se han jerarquizado (Padrón \& Alba, 2019), dando más peso a las ciencias técnicas y exactas y menos a las ciencias sociales. El debate no es nuevo, y la brecha existente entre las distintas disciplinas y profesionales que se dedican a estos campos ya fue expuesta en 1959 por Charles Percy, que se posicionó del lado de la ciencia al entender que era el vehículo de los avances, y advirtió de la brecha existente entre científicos e intelectuales. Su concepto de "las dos culturas", fue ampliamente utilizado y referenciado posteriormente (Brockman, 1996).

No obstante, a día de hoy en la tradición anglosajona es muy común la división entre ciencias "duras" y ciencias "blandas" (Meneses, 2016), diferenciando así lo que en nuestro entorno más cercano también se han venido llamando "las ciencias" y "las letras" (López-Maroto, 2020). Algunos autores rechazan esta dicotomía al considerar que no ha de ponerse en duda el carácter científico de las ciencias sociales (Gil Antón, 2004). Al fin y al cabo, tanto las ciencias naturales como las sociales analizan datos empíricos utilizando métodos cuantitativos y cualitativos, siendo el objeto de análisis la principal diferencia (Priest, 2014:63), que en el caso de las ciencias sociales es más abstracto y cambiante.

A pesar de que, tal como señala Nussbaum (2010), el desarrollo económico y tecnológico actual hace que se priorice la tecnología, el saber también tiene que contribuir al conocimiento de la sociedad y sus problemáticas, para aumentar la calidad de vida de las personas (Nussbaum, 2010; Marrero, 2018).

Tal y como se ha señalado, esta discusión también se ha trasladado al área del periodismo científico, porque la falta de una reflexión y definición más generalizada de lo que se entiende por ciencia hace que sea más difícil analizar la cobertura de los medios de comunicación sobre las ciencias sociales y las humanidades (Cassady, 2021). ¿Qué es el periodismo científico o el periodismo sobre ciencia? Wormer lo define como aquel que emplea "resultados, instituciones y procesos en ciencia, tecnología y salud" (Rosen, 2017: 229). Se trataría de lo que el mismo autor califica como la definición clásica de la ciencia y trabajaría, sobre todo, con hallazgos, proyectos y conferencias científicas. Sin embargo, una segunda definición más amplia también incluiría temas del día a día o temas generales, si para ello el periodista precisa hacer alguna referencia a un científico o a un estudio (Wormer, 2008). Por lo tanto, tal y como señalaban Summ y Volpers (2016), en esta segunda definición los contenidos sobre ciencias sociales tienen mayor cabida, por 
lo que resulta más efectiva a la hora de enfrentarse a una investigación en la que se tomen en cuenta.

Otros autores han puesto, sin embargo, el foco en las funciones y objetivos del periodismo científico (Nelkin, 1995; Calvo Hernando, 2001; Miller, 2010) y hay quien se ha detenido en los métodos de los periodistas que escriben sobre ciencia, así como en las herramientas lingüísticas que se emplean (Elías, 2008). La mayoría de estos últimos estudios también se refieren, aunque más implícitamente, a las ramas científicas denominadas "duras", pero muchas de las características y procedimientos descritos dan la oportunidad de incluir también las noticias sobre ciencias sociales o humanidades. Dado el debate que existente, algunos autores han incidido en la necesidad de ampliar la definición misma de la "ciencia" para analizar también las ciencias sociales (Davies \& Horst, 2016). En este contexto, proponen la utilización del concepto "comunicación de la investigación" (Cassidy, 2021).

Sin una definición unificada y consensuada, los investigadores que pretenden analizar todas las áreas del conocimiento científico han optado por realizar sus propias delimitaciones. Así, Meso y Díaz Noci (2002), en su estudio sobre las revistas científicas digitales, optaron por definir la comunicación científica como "todo tipo de puesta a disposición del publico, más o menos amplio, de la comunidad científica o de cualquiera mínimamente interesado, de los conocimientos que se producen en el mundo académico" (2002:607). Consideraron que esta visión más amplia les ofrecía la oportunidad de conocer más certeramente el fenómeno. En esta línea, Olvera-Lobo y López Pérez (2015:769) seleccionan como noticias científicas aquellas que incluyen resultados de investigaciones en todas las áreas de conocimiento o abordan resultados relacionados con las ciencias aplicadas, la ingeniería o la medicina, así como las noticias o los artículos donde la ciencia o su metodología son la base de la argumentación.

Estas delimitaciones del objeto, aunque prácticas, no resuelven los debates en torno a la definición de la ciencia, ni tampoco lo hacen otros estudios que han optado por limitar las áreas analizadas (Summ \& Volpers, 2016) o han limitado su examen a actividades científicas concretas (Meneses, 2016), evitando así el debate en torno a la definición de la noticia científica.

En cualquier caso, y a pesar de que todavía son una minoría, varios estudios sí han incluido las ciencias sociales y las humanidades en sus investigaciones (Dunwoody, 1984; Haslam \& Bryman, 1994; Cassidy, 2008; Summ \& Volpers, 2016; Meneses, 2016; Segado et al., 2018), siendo la psicología la que, tradicionalmente, más interés ha suscitado (Cassidy, 2021). Los datos obtenidos por este tipo de estudios son, sin embargo, contradictorios. Mientras algunos indican que la presencia de las ciencias sociales y humanidades es significativa (Cassidy, 2008); otros concluyen que es residual (Segado et al., 2018). A este respecto, una definición consensuada de la noticia científica quizás podría ayudar a unificar metodologías y a analizar con más rigor la presencia de las ciencias sociales y las humanidades en los contenidos periodísticos, así como el tratamiento diferenciado que se ofrece a los científicos y científicas de las disciplinas sociales (Haslam \& Bryman, 1994).

En este contexto, el presente estudio tiene como objetivo analizar la presencia de las ciencias sociales y las humanidades en las informaciones de los medios de comunicación online, así como estudiar las características que los contenidos sobre estas presentan. Por lo tanto, las preguntas de investigación que se plantea y pretende responder este trabajo son las siguientes:

- Q1: ¿Qué porcentaje de contenidos corresponden a las ciencias sociales y las humanidades?

- Q2: ¿Qué disciplinas son las más representadas?

- Q3: ¿En qué secciones se publican los contenidos sobre ciencias sociales y humanidades?

- Q4: ¿Qué géneros informativos son los que más se emplean?

- Q5: ¿Qué disciplinas generan más interacción de los lectores?

A este respecto, a pesar de que el objeto de la investigación pretende centrarse en las ciencias sociales y las humanidades, la recogida y el posterior análisis de los datos también ha estudiado las características de los textos sobre ciencias experimentales y aplicadas, entendiendo que estos 
datos ayudan a contextualizar mejor la realidad analizada y a enriquecer los resultados, ya que posibilitan un examen comparado.

\section{Metodología}

El objetivo de esta investigación ha sido analizar las características de las noticias científicas que se publican en las versiones online de El País (www.elpais.es), The Guardian (www.theguardian.com) y Público (www.publico.pt) y comparar así las similitudes y las diferencias que existen entre España, Gran Bretaña y Portugal. Los tres diarios se encuentran entre los cibermedios líderes de cada uno de sus países, entre los medios de referencia según los datos de Comscore. Para ello, se ha analizado la temática de cada noticia, la sección en la que se publica, el género periodístico empleado, así como la interacción resultante. La muestra de cabeceras para realizar el estudio está formada por las ediciones digitales, todos ellos referentes en sus ámbitos geográficos, y el periodo analizado es el año 2020.

El método empleado es el análisis de contenido, una técnica utilizada de manera frecuente en la investigación sobre los medios de comunicación y también sobre el periodismo científico, y cuyo uso se ha intensificado debido a la mayor disposición de información textual, fundamentalmente a través de internet (Andreu, 2001). Se trata, en efecto, de una técnica que permite analizar los textos de forma sistemática, objetiva y cuantitativa (Wimmer \& Dominick, 1996) y que posibilita, mediante los datos obtenidos, realizar inferencias reproducibles y válidas que pueden aplicarse en el contexto (Kippendorff, 1990).

Para extraer la muestra, se optó por la técnica de la semana compuesta, un método avalado en la literatura científica y que establece que para ofrecer estimaciones confiables sobre ediciones periodísticas de un año es suficiente con el análisis de dos semanas compuestas (Riffe et al., 1993). En este caso, se realizó la captura de la muestra durante el primer y tercer trimestre del año 2020, más concretamente, entre los días 27 de enero y 15 de marzo y 22 de junio y 16 de agosto.

Ha resultado necesario concretar cuáles son las cualidades definitorias que se establecen para determinar qué es un texto de ciencia. De cara a este análisis, se han seleccionado aquellas piezas periodísticas que en el titular o en el sumario -si es que lo hubiera- o en el lead, hacen alusión a una investigación o estudio, así como las que tienen como eje las declaraciones u opiniones de actores científicos, a pesar de que estos elementos no sean presentados nominalmente en estos apartados. Es decir, para que una pieza sea considerada de ciencias sociales o humanidades se le ha exigido que cite investigadores o estudios del tema en la cabecera o el lead, a pesar de que la alusión precisa la realice posteriormente en el cuerpo de la unidad informativa.

Como el objetivo ha sido analizar los contenidos de ciencia en su totalidad, indistintamente que estos se incluyesen en las secciones específicas o no, la búsqueda de contenidos ha abarcado todos los apartados presentados en la portada de cada medio.

Para el proceso de registro y la posterior codificación se ha tomado como referencia la nomenclatura internacional de la Unesco para los campos de la ciencia y la tecnología. Esta clasificación, creada en 1973, se ha empleado como guía por muchas organizaciones internacionales $\mathrm{y}$, a pesar de mostrar algunas deficiencias, tales como la obsolescencia o las carencias que representa a nivel de representatividad y usabilidad, sigue siendo un modelo general, estándar y reutilizable (Ruiz-Martínez et al., 2014: 388). Este código clasifica las áreas del saber en 24 campos $^{1}$ a los que se ha optado por añadir uno más: ciencias de la comunicación. Esta decisión metodológica responde a la propuesta realizada por algunos estudios (Marzal-Felici et

\footnotetext{
${ }^{1}$ Lógica, matemáticas, astronomía y astrofísica, física, química, ciencias de la vida, ciencias de la tierra y el espacio, ciencias agrónomas, ciencias médicas, ciencias tecnológicas, antropología, demografía, ciencias económicas, geografía, historia, ciencias jurídicas, lingüística, pedagogía, ciencia política, psicología, ciencias de las artes y las letras, sociología, ética y filosofía.
} 
al., 2016; García-García, 2007) que inciden en la necesidad de incorporar este campo, que actualmente se encuentra subsumido en otras áreas (Moragas, 2013). Por lo tanto, se han tenido en cuenta un total de 25 campos.

Con estos requisitos, se han registrado un total de 208 piezas en los medios analizados $(\mathrm{n}=208)$, con una distribución desigual entre las distintas cabeceras. La página web de Público (www.publico.pt) es la que más contenidos de ciencia ha publicado $(45,2 \%)$, seguida de la de El País $(31,7 \%)$ y de la de The Guardian $(23,1 \%)$.

\section{Resultados}

Las piezas periodísticas que guardan relación con las ciencias sociales y las humanidades representan el 33,65\% del total de textos que integran la muestra, mientras que las ciencias experimentales y aplicadas suponen el $66,35 \%$ restante (Figura 1). La cifra varía visiblemente según el medio analizado. (Figura 1). El País es el medio que más artículos ha publicado sobre ciencias sociales y humanidades $(40,00 \%)$, seguido por Público $(37,14 \%)$ y, por último, The Guardian (22,85\%). No obstante, si se atiende a los porcentajes de estas disciplinas en el cómputo de cada medio, El País sigue siendo el que mayor porcentaje muestra $(45,45 \%)$, pero detrás se sitúan The Guardian (33,33\%) y Público (27,65\%).

Figura 1. Presencia de las ciencias sociales y las humanidades en las informaciones sobre ciencia (total y por medio de comunicación).

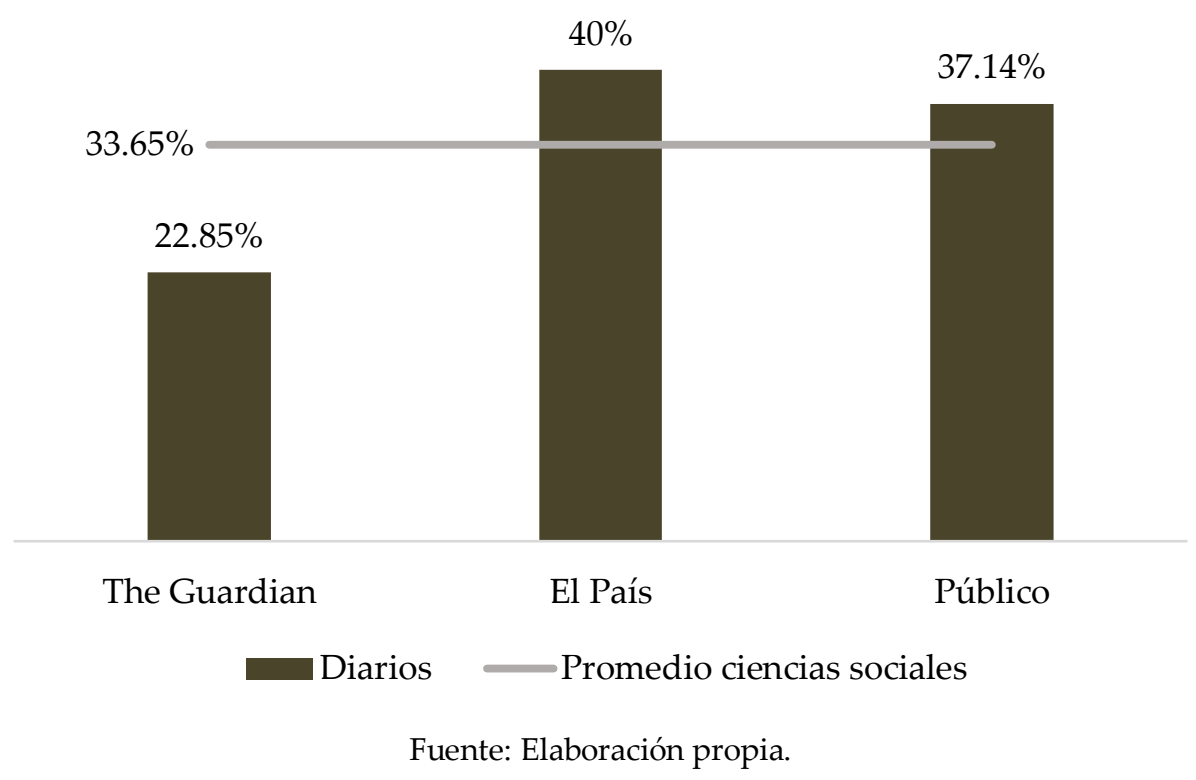

Dentro de los temas de ciencias sociales y humanidades ( $\mathrm{n}=70)$, la historia $(17,14 \%)$ y la economía $(14,29 \%)$ son las disciplinas con mayor representación, seguidas de contenidos relacionados con la pedagogía (11,43\%) y con el derecho, la psicología y la sociología (Figura 2). En menor medida, la muestra analizada también ha incluido contenidos relacionados con las ciencias de la comunicación, las ciencias políticas y la filosofía, la antropología, el arte y la lingüística. 
Figura 2. Presencia de las disciplinas de las ciencias sociales y humanidades (en \%).

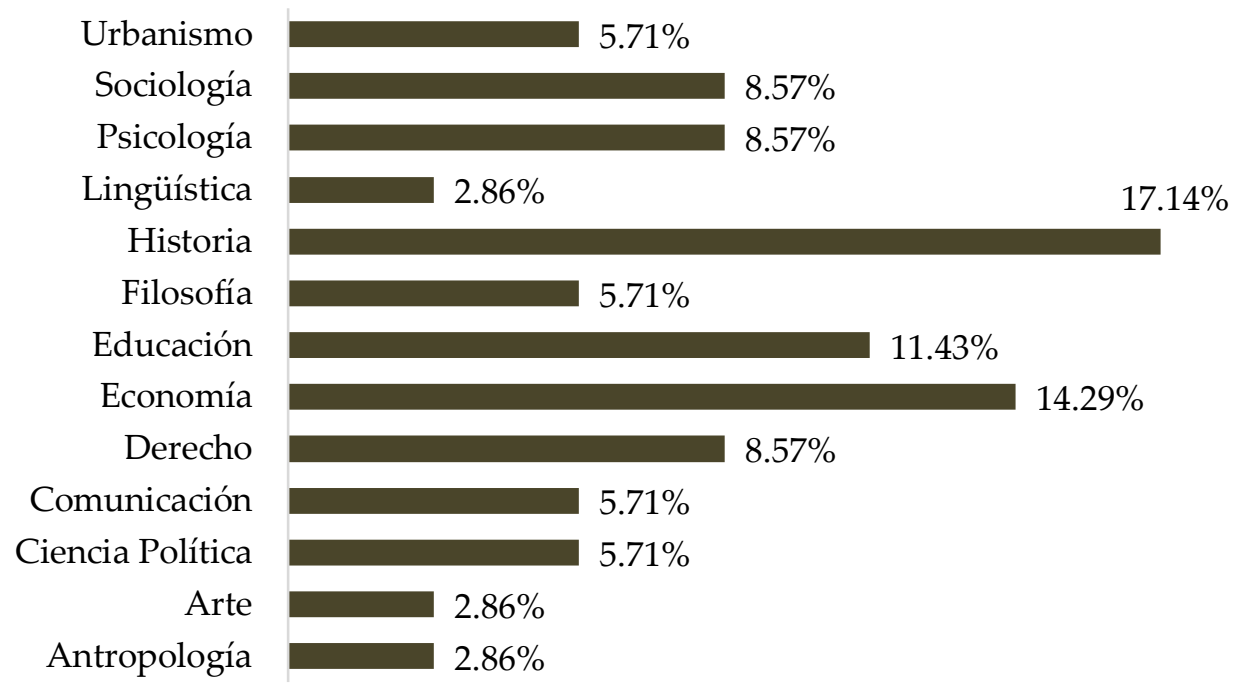

Fuente: Elaboración propia.

En cuanto a las ciencias experimentales y aplicadas, del total de ítems analizados ( $\mathrm{n}=138)$, sin duda, los temas sanitarios, tanto desde el ámbito de las ciencias de la salud como de las ciencias de vida, son los que más atención reciben, ya que representan el 73,91\% de los contenidos dentro de las ciencias "duras" y el 36,50\% de todas las unidades analizadas.

Las ciencias de la salud y las ciencias de la vida acaparan la mayor parte de la atención de los medios de comunicación analizados. Les siguen, ya a bastante distancia, la astronomía y la astrofísica $(7,25 \%)$, las ciencias del espacio y la tierra $(5,80 \%)$, la tecnología $(5,80 \%)$, las matemáticas $(4,35 \%)$ y la física $(1,44 \%)$ (Figura 3).

Figura 3. Disciplinas de las ciencias experimentales y aplicadas (en \%).

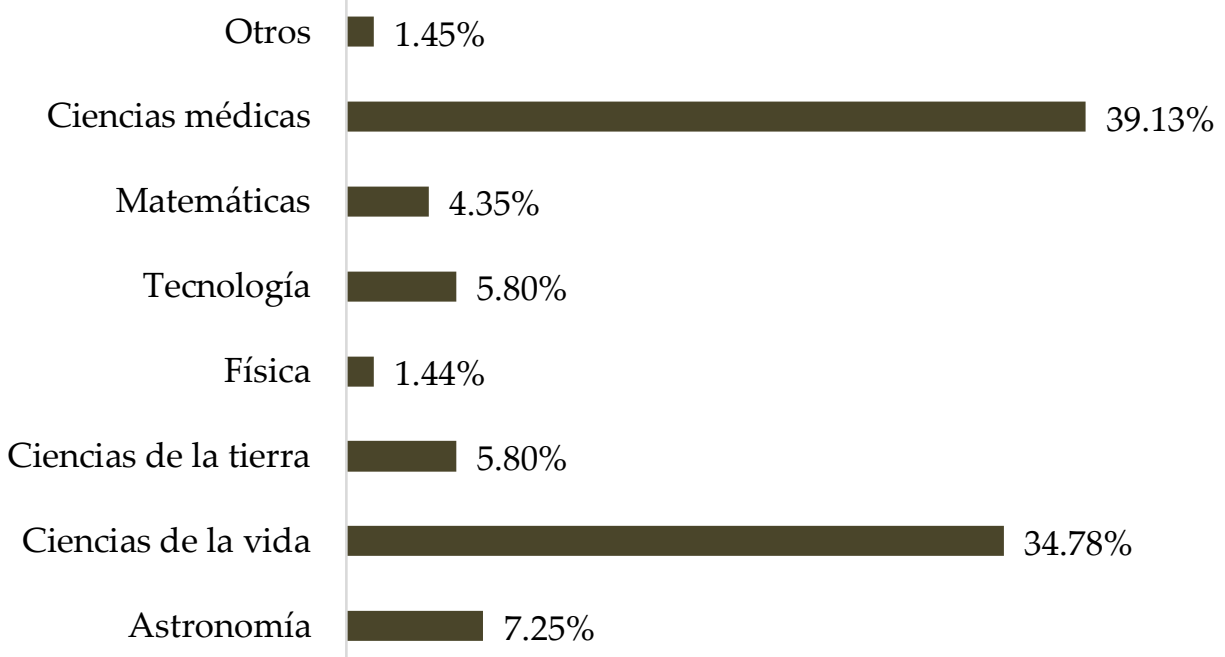

Fuente: Elaboración propia.

Si nos fijamos en las secciones en las que se publican estas informaciones, los datos indican que los contenidos científicos sobre las ciencias sociales y las humanidades tienen una importante presencia fuera de las secciones tradicionales, es decir, en los distintos suplementos, tanto 
dominicales como temáticos. Más de un tercio de los artículos analizados $(34,28 \%)$ se han publicado en este tipo de apartados. Dentro de las secciones más habituales, Internacional es la que más informaciones engloba (20\%), seguida de Ciencia (14,28\%), Sociedad (11,43\%), Economía $(8,57 \%)$, Cultura $(5,72 \%)$, Política $(2,86 \%)$ y Local $(2,86)$ (Figura 4$)$.

Para valorar esta circunstancia es interesante analizar comparativamente qué es lo que sucede con los contenidos sobre las ciencias experimentales y aplicadas. En este caso, más de la mitad de los contenidos se publican en el apartado de ciencia o aquellas que contienen temas tecnológicos o medioambientales. El porcentaje de contenidos publicados en los suplementos es del 17,39\% y detrás se colocan las secciones de Sociedad e Internacional, ambas con un 11,59\%.

Figura 4. Secciones en las que se publican informaciones sobre ciencia (en \%).

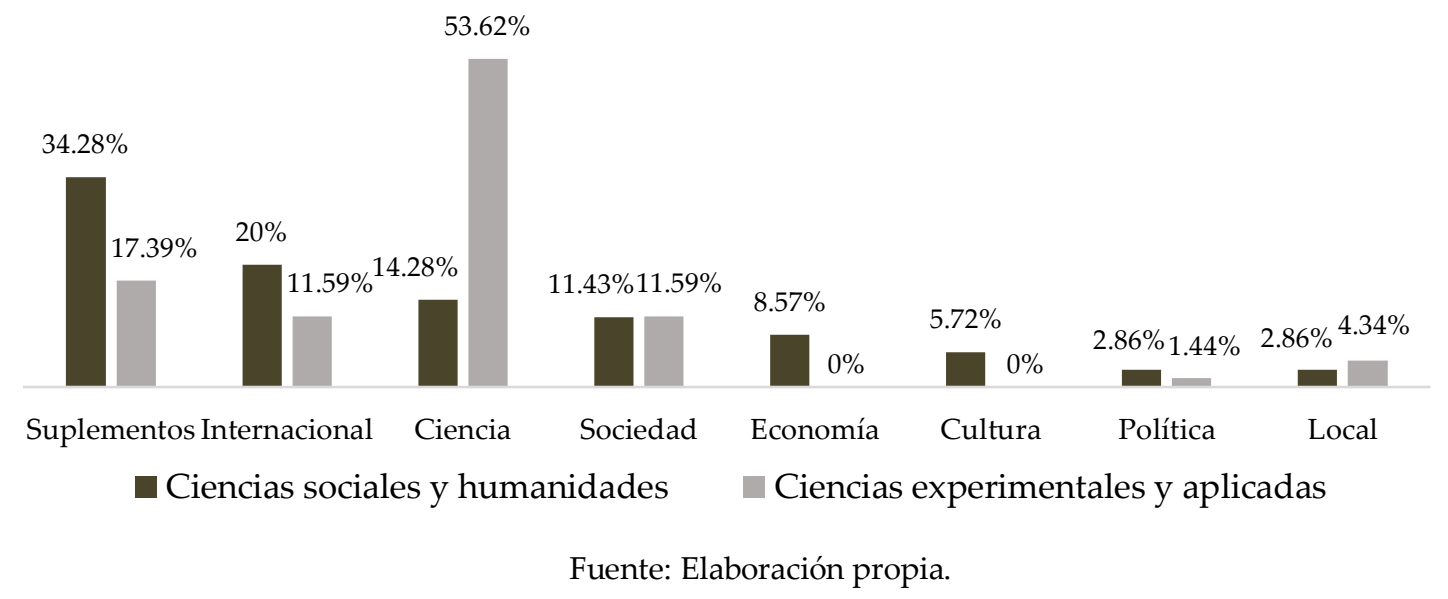

Más allá de los datos generales, se aprecian diferencias entre los distintos medios. Público es el medio que, si se tienen en cuenta todas las áreas científicas, registra un porcentaje más alto en la sección de ciencia, ya que casi la mitad $(48,93 \%)$ de los contenidos de este medio provienen de esta sección. El porcentaje de El País (42,42\%) también es significativo, no así el de The Guardian que baja hasta el 20,83\%. En este último medio destaca principalmente la sección de Internacional, ya que en ella se incluyen el $45,83 \%$ de las informaciones analizadas. Si observamos, las ciencias sociales y las humanidades, el porcentaje de contenidos que se publican en la sección de ciencia baja significativamente y en el caso de The Guardian, ningún contenido de estas áreas se presenta en esta sección específica. El País es el medio que más artículos de ciencias sociales incluye en la sección de ciencia, un 28,58\% una cifra que en Público disminuye hasta el 7,69\%. En todos los medios el peso de los suplementos es considerable: del 35,71\% en El País, 30,70 \% en Público y del 25,00\% en The Guardian. Destaca, sin embargo, la presencia de estos contenidos en la sección de Sociedad: 15,38\% en Público; del 14,28\% en El País y del 12,50\% en The Guardian.

También se percibe un uso diferenciado de los géneros periodísticos. Si bien, la noticia es el género más empleado en temas sobre ciencias sociales y humanidades, con un $40 \%$, el porcentaje de reportajes y entrevistas es significativo, con un $30 \%$ y un $26,70 \%$ respectivamente. Mientras tanto, en el área de las ciencias experimentales y aplicadas el porcentaje de noticias es mayor y alcanza el 68,12\% (Figura 5). Esta circunstancia se debe principalmente a que en estas últimas áreas predominan las noticias sobre hallazgos científicos, mientras que en el caso de las ciencias sociales es más habitual la alusión a investigaciones e investigadores que valoran o enriquecen los temas a tratar, a pesar de que, tal y como señaló McCall (1988), los medios piden a los científicos sociales generalizar los resultados de su investigación y comentar temas que no han sido abordados en su estudio. 
Figura 5. Secciones en las que se publican informaciones sobre ciencia (en \%).

Ciencias Sociales y Humanidades

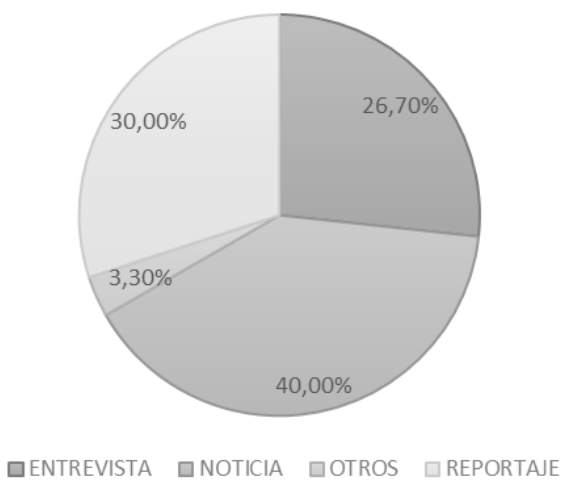

Ciencias experimentales y aplicadas

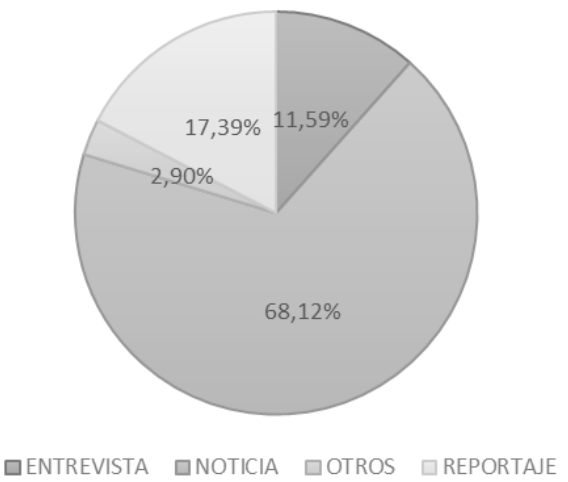

Fuente: Elaboración propia.

Por último, en lo que se refiere a la interacción de los lectores con los contenidos sobre ciencia, el promedio de comentarios ha sido de 8 por cada información. Si nos centramos en las 20 informaciones que más comentarios han logrado, podemos observar que las ciencias experimentales y aplicadas destacan por encima de las ciencias sociales y las humanidades (Figura 6). Además, los temas sanitarios muestran una prevalencia significativa; diez de los veinte temas más comentados se refieren a distintas áreas relacionadas con la salud. Por el contrario, tan solo cinco de los veinte contenidos con más comentarios pertenecen a las ciencias sociales y humanidades. Entre los medios analizados, The Guardian es el medio en el que los lectores más interactúan, con cinco de las seis piezas más comentadas.

Figura 6. Número de interacciones de las noticias más comentadas, por disciplinas (en \%).

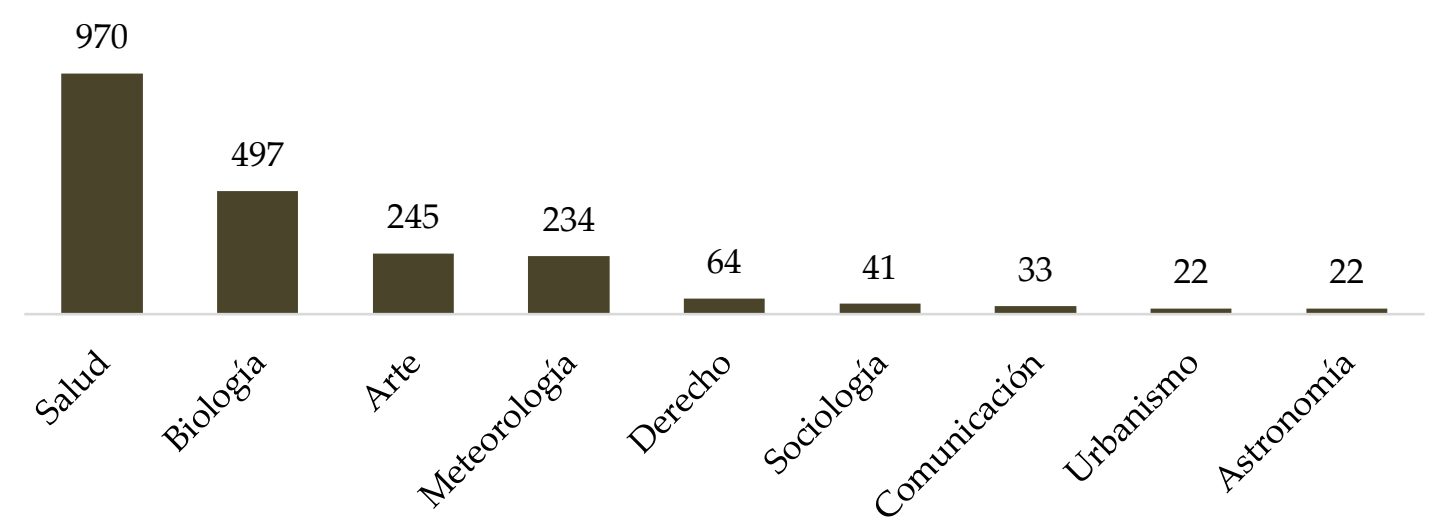

Fuente: Elaboración propia.

\section{Conclusiones}

El estudio sobre la presencia de las ciencias sociales en tres grandes medios de comunicación de referencia -El País (España), Público (Portugal) y The Guardian (Reino Unido) permite concluir que la presencia de las ciencias sociales y las humanidades entre las informaciones científicas no es tan marginal como señalaban otros estudios (Segado-Boj, 2018). En total, las informaciones que toman como base investigaciones $u$ opiniones de investigadores de estas disciplinas alcanza el 33,65\% del total de informaciones sobre ciencia. El reparto por disciplinas 
es relativamente disperso, aunque la historia y la economía suman casi una de cada tres informaciones del área, en contraste con otras investigaciones existentes (Cassidy, 2021).

Por medios, la menor presencia en The Guardian puede guardar relación con que la tradicional división entre ciencias "duras" y "blandas" sea más acusada en el ámbito anglosajón y que dentro del ámbito periodístico, incluso, las hayan considerado "garbage science" (Dunwoody, 1984). No obstante, los porcentajes obtenidos en los tres medios obligan a reflexionar y a plantearse la necesidad de ampliar la mirada a la hora de analizar los temas de ciencia en los medios de comunicación.

Es preciso matizar que el año 2020 ha estado fuertemente marcado por la crisis sanitaria del Covid-19 y que este hecho ha marcado también la agenda mediática, en la que los temas médicos han adquirido una gran relevancia, tal y como se ha podido comprobar también en este estudio. Los profesionales de la comunicación han tenido que hacer frente a la dificultad de valorar la situación (Dunwoody, 2020; Catalán-Matamoros, 2020) y, por lo tanto, la presencia de estudios y científicos sanitarios y del área biomédica ha sido importante. Está por ver, si la inclusión de científicos como fuentes autorizadas para valorar los acontecimientos se extiende en el tiempo y a distintos temas, o se trata de tendencia pasajera impuesta por la actual situación marcada por la incertidumbre y la proliferación de noticias falsas sobre la pandemia (Aguado \& Bernaola, 2020; Elías, 2020). No obstante, estos datos no hacen más que confirmar la tendencia al alza de los temas médicos (Olvera-Lobo \& López Pérez, 2015), una inclinación que algunos autores han descrito como la "medicalización" de las noticias científicas (Bauer, 2003), a pesar de que, en algunos casos estas presenten un componente social y se centren más en los beneficios personales y sociales de la investigación médica, que en las evidencias y características metodológicas de los avances realizados.

En segundo lugar, puede también concluirse que las ciencias sociales también siguen pautas diferentes tanto en lo que a secciones se refiere como a la utilización de distintos géneros periodísticos. A primera vista, las secciones guardan más relación con la presentación de los textos que con su contenido, pero la división de artículos periodísticos en diferentes apartados temáticos también puede tener repercusiones en las particularidades del texto (Olvera-Lobo \& López Pérez, 2015) así como en las fuentes empleadas (Segado et al., 2018). Así, las informaciones sobre ciencias sociales se publican más en los suplementos y en un porcentaje menor en las secciones de Internacional, Sociedad, Ciencia y Economía. En oposición, la mitad de los contenidos sobre las ciencias "duras" encuentran su lugar en la sección de Ciencia, lo que sugiere que, a pesar de que en todos los casos se citen estudios o declaraciones de profesionales de la ciencia, se atribuye un carácter más científico a aquellos que se refieren a este tipo de disciplinas. A primera vista, las secciones guardan más relación con la presentación de los textos que con su contenido, pero la división de artículos periodísticos en diferentes apartados temáticos también puede tener repercusiones en las particularidades del texto (Olvera-Lobo \& López Pérez, 2015) así como en las fuentes empleadas (Segado et al., 2018).

En cuanto a los géneros informativos, el empleo de entrevistas y reportajes es mayor también en el caso de las ciencias sociales, lo cual podría sugerir que los medios analizados dan una relevancia mayor a este tipo de temáticas. No obstante, el análisis de las informaciones revela que en el caso de las ciencias sociales es más común la alusión a un estudio o a las declaraciones del científico o la científica en calidad de fuente documental o personal que valora o enriquece un tema; en el caso de las ciencias "duras" muchos de los contenidos son noticias sobre la presentación de resultados de una investigación. Por tanto, puede deducirse que los resultados de las investigaciones de las áreas CTIM son considerados más noticiosos, mientras que las investigaciones sociales se utilizan en mayor medida como fuente de autoridad.

Por lo tanto, a la vista de los resultados obtenidos y en relación a la interrogante que plantea el estudio, las ciencias sociales y humanas no están desaparecidas de los medios de comunicación. Quizás, tampoco se puede afirmar contundentemente que estén escondidas, ya que los científicos y las investigaciones de algunas ramas del saber encuentran su espacio en aquellas áreas a las que 
se asocian con mayor naturalidad. Ejemplo de ello son los temas relacionados con la economía, publicados, casi sin excepción, en la sección de Economía.

El hecho de que, tal y como se ha indicado, muchas de las piezas sobre ciencias sociales y humanidades se presenten en los suplementos o fuera de las secciones tradicionales, puede guardar relación con el diferenciado tratamiento de las investigaciones en estas áreas, quizás más asequibles para los profesionales de los medios. El tema se presta, por lo tanto, a que futuros estudios analicen la propia esencia de las secciones específicas de ciencia y valorar en qué medida responden a la dificultad de emplazar ciertos temas en las secciones tradicionales o si su desarrollo se corresponde, por lo contrario, con una intención de visibilizar y significar a ciertos temas científicos.

La citada es una de las vías de investigación que abre este estudio, la otra procede, precisamente, de la principal limitación de este estudio, pues no existe una definición unificada acerca de las noticias de ciencia que incluyan a las ciencias sociales o las humanidades, por lo que los distintos estudios que se han centrado en esta cuestión han optado por utilizar una definición creada ad hoc para su desarrollo, lo que dificulta su comparación.

\section{Referencias}

Aguado-Guadalupe, G., \& Bernaola-Serrano, I. (2020). Verificación en la infodemia de la Covid-19. El caso Newtral. Revista Latina, 78, 289-308. doi:10.4185/RLCS-2020-1478. https://doi.org/10.4185/RLCS-2020-1478

Alcíbar, M. (2004). La divulgación mediática de la ciencia y la tecnología como recontextualización discursiva. Anàlisi: Quaderns de comunicació i cultura, 31, 43-70.

Andreu, Jaime (2002). Las técnicas de análisis de contenido: una revisión actualizada. Fundación Centro de Estudios Andaluces.

Bauer, M. W., Howard, S., Romo Ramos, Y. J., Massarani, L., \& Amorim, L. (2013). Global science journalism report: working conditions $\mathcal{E}$ practices, professional ethos and future expectations. Science and Development Network. http://eprints.lse.ac.uk/id/eprint/48051

Brockman, J., \& Scott, A. (1996). The Third Culture: Beyond the Scientific Revolution. American Journal of Physics, 64(3), 348-349. https://doi.org/10.1119/1.18425

Brossard, D. (2013). New media landscapes and the science information consumer. Proceedings of the National Academy of Sciences, 110(3), 14096-14101. https://doi.org/10.1073/pnas.1212744110

Brossard, D., \& Scheufele, D. A. (2013). Science, new media, and the public. Science, 339(6115), 40-41. https://doi.org/10.1126/science.1232329

Bucchi, M., \& Mazzolini, R. G. (2003). Big science, little news: science coverage in the Italian daily press, 1946-1997. Public understanding of science, 12(1), 7-24. https://doi.org/10.1177/0963662503012001413

Casero Ripollés, A. (2020). La COVID-19 en el periodismo: un impacto ambivalente. Revista de la Asociación Española de Investigación de la Comunicación, 7(14), 2-26. https://doi.org/10.24137/raeic.7.14.1

Cassidy, A. (2008). Communicating the Social Sciences. In M. Bucchi \& and B. Trench (Eds.), Handbook of Public Communication of Science and Technology (pp. 225-236). Routledge.

Cassidy, A. (2021). Communicating the Social Sciences. In M. Bucchi \& and B. Trench (Eds.), Handbook of Public Communication of Science and Technology (pp. 1948-1954). Routledge. 
Cortiñas-Rovira, S., \& Alonso-Marcos, F. (2014). La decadencia de la sección deficiencia en los medios $\begin{array}{llll}\text { tradicionales. } & \text { Prisma Social, } & \text { 402-435. }\end{array}$ http://www.isdfundacion.org/publicaciones/revista/numeros/12/secciones/tematica/t-12-decadenciamedios.html

Daltoé, A. (2003). Promessas, desafios e ameaças das tecnologias digitais. Bocc: biblioteca on-line de ciências da comunicação. http://www.bocc.ubi.pt/pag/daltoe-andrelise-promessas-desafios-tecnologias-digitais.pdf

Davies, S. R., \& Horst, M. (Eds.). (2016). Science Communication: Culture, Identity and Citizenship. Palgrave Macmillan Springer International.

De Semir, V. (2010). El «mutatis mutandis» de la comunicación científica en la era de internet. ArtefaCToS. Revista de estudios sobre la ciencia y la tecnología, 3(1), 49-79. https://revistas.usal.es/index.php/artefactos/article/view/8429

De Semir, V. (2015). Decir la ciencia. Divulgación y periodismo científico de Galileo a Twitter. Edicions Universitat Barcelona.

Deuze, M. (2001). Online journalism: Modelling the first generation of news media on the World Wide Web. First Monday, 6(10). https://doi.org/10.5210/fm.v6i10.893

Dunwoody, S. (1984). Mass media coverage of the Social Sciences: Some new answers to old questions. Ecquid Novi, 5(2), 83-92. https://doi.org/10.1080/02560054.1984.9652936

Dunwoody, S. (2008). Science journalism. In M. Bucchi \& B. Trench (Eds.), Handbook of public communication on science and technology (pp. 15-26). Routledge.

Dunwoody, S. (2020). Science Journalism and Pandemic Uncertainty. Media and Communication, 8(2), 471474. http://dx.doi.org/10.17645/mac.v8i2.3224

Elías, C. (2008). El Periodismo científico como paradigma de la «noticia acatamiento»: una demostración desde las fuentes y una alerta de sus peligros. Periodística: revista acadèmica, 2008, 81-93. https://www.raco.cat/index.php/Periodistica/article/view/245699

Elías, C., \& Catalan-Matamoros, D. (2020). Coronavirus in Spain: Fear of 'Official' Fake News Boosts WhatsApp and Alternative Sources. Media and Communication, 8(2), 462-466. http://dx.doi.org/10.17645/mac.v8i2.3217

Fernández de Lis, P. (2014). Ciencia y periodismo en la red. Quaderns de la Fundació Dr. Antoni Esteve, 28, 1519.

Francescutti, P. (2018). La visibilidad de las científicas españolas. Fundación Dr. Antoni Esteve.

Franquet, R., Ramajo, N., \& Luzón, V. (2007). La información en los principales medios de comunicación online: estudiar la representación de género. Zer: revista de estudios de comunicación = komunikazio ikasketen aldizkaria, 12(22), 267-282. https://ojs.ehu.eus/index.php/Zer/article/view/3688

García García, F. (2007). Los medios de comunicación y los códigos de la UNESCO. Revista ICONO14 Revista Científica de Comunicación y Tecnologías Emergentes, 5(1), 384-396. https://doi.org/10.7195/ri14.v5i1.383

Gil Antón, M. (2004). ¿Ciencias duras y ciencias blandas? Una falsa dicotomía. Contaduría y Administración, 213, 151-164.

Granado, A. (2011). Slaves to journals, serfs to the web: The use of the Internet innewsgathering among European science journalists. Journalism, 12(7), 794-813. https://doi.org/10.1177/1464884911412702

Haslam, C., \& Bryman, A. (Eds.). (1994). Social scientists meet the media. Routledge. 
Hernando, M. C. (1984). Periodismo científico. Revista Comunicación y Medios, 4, 79-105.

López-Cantos, F., Cortiñas-Rovira, S., \& Itatí Rodríguez, M. (2020). Comunicación del Conocimiento Científico en la Era de la Postverdad. Retos y Oportunidades. Revista Prisma Social, 31, 1-5. https://revistaprismasocial.es/article/view/4077

Lopez-Maroto. S. (2020). Los textos científicos latinos como punto de partida para un entendimiento entre ciencias y letras: una propuesta didáctica. Estudios clásicos, 157, 167-196. https://doi.org/10.48232/eclas.157.07

Marrero, A. (2018). Las jerarquías de las disciplinas y del conocimiento en uruguay. El lugar de las ciencias sociales en los incentivos a la investigación en el marco de la sociedad del conocimiento. International Journal Education and Teaching, 1(2), 23-46. https://doi.org/10.31692/2595-2498.v1i2.43

Marzal-Felici, J., García Jiménez, A., \& Humanes, M. L. (2016). Análisis y reformulación de la organización del conocimiento en las Ciencias de la Comunicación: aplicación para la codificación UNESCO. Revista General de Información y Documentación, 26(1), 65-79. https://doi.org/10.5209/rev_RGID.2016.v26.n1.53049

McCall, R. B. (1988). Science and the press: Like oil and water? American Psychologist, 43(2), 87-94. https://doi.org/10.1037/0003-066X.43.2.87

Meneses, M. D. (2016). ¿Relegan los informativos audiovisuales en España la investigación en Ciencias Humanas y Sociales? 'Las otras ciencias'. Revista Española de Documentación Científica, 39(3), 1-14. doi: https://doi.org/10.3989/redc.2016.3.1326

Meso Ayerdi, K., \& Díaz Noci, J. (2002). Periodismo científico en el ciberespacio: la información académica al encuentro de la tecnología digital. Mediatika, 8, 605-629.

Molek-Kozakowska, K. (2017). Stylistic Analysis of Headlines in Science Journalism: A Case Study of New Scientist. Public understanding of science, 26(8), 894-907.

Moragas Spà, M. (2011). Las asociaciones de investigación de la comunicación. Funciones y retos. Actas del IV Congreso Internacional de la AE-IC. http://www.ae-ic.org/upload/ponencia_mdemoraas_esp.pdf

Nelkin, D. (1995). Selling science: How the press covers science and technology. W. H. Freeman.

Nussbaum, M. (2010). Sin fines de lucro: por qué la democracia necesita de las humanidades. Katz editories.

Olvera-Lobo, M.D., \& López-Pérez, L. (2015). Periodismo científico: la homogeneización de la información del papel a internet. Journal of Science Communication, 14(3), 2-12.

Padrón, J. L., \& Alba, D. (2019). De las "Ciencias duras" y las "Ciencias blandas". Apuntes sobre las "Dos culturas". Revista Caribeña de Ciencias Sociales, (octubre 2019).

Peters H. P., Dunwoody S., Allgaier J., Lo Y.Y., \& Brossard D. (2014). Public communication of science 2.0: Is the communication of science via the "new media" online a genuine transformation or old wine in new bottles? EMBO reports, 15(7), 749-753. https://doi.org/10.15252/embr.201438979

Priest, S. (2014). ¿Qué tienen de científico las Ciencias Sociales? La complejidad de medir el comportamiento humano. Mètode Science Studies Journal, 84, 57-63. https://doi.org/10.7203/metode.84.3923

Riffe, D., Aust, C. F., \& Lacy, S. R. (1993). The effectiveness of random, consecutive day and constructed week sampling in newspaper content analysis. Journalism quarterly, 70(1), 133-139. https://doi.org/10.1177/107769909307000115

Rosen, C. (2017). La función social en el periodismo de ciencias. Una exploración de las percepciones de los periodistas en la Argentina. In R. Casas \& T. Pérez-Bustos, T. (Eds.), Ciencia, tecnología y sociedad en América Latina: La mirada de las nuevas generaciones (pp. 229-256). CLACSO. 
Ruiz-Martínez, J. M., Baños-Moreno, M. J., \& Martínez-Béjar, R. (2014). Nomenclatura Unesco: evolución, alcance y reutilización en clave ontológica para la descripción de perfiles científicos. Profesional de la Información, 23(4), 383-392. https://doi.org/10.3145/epi.2014.jul.06

Russell, C. (2010). Covering controversial science: Improving reporting on science and public policy. In D. Kennedy \& G. Overholser (Eds.), Science and the Media (pp. 13-43). American Academy of Arts \& Sciences.

Salaverría, R. (2005). Redacción periodística en internet. Eunsa.

Schäfer, M. S. (2017). How changing media structures are affecting science news coverage. In K. Hall, D. Kahan \& D. A. Scheufele (Eds.), The Oxford Handbook of the Science of Science Communication, (pp 51-57). Oxford University Press.

Segado, F., Chaparro, M. A., \& Díaz, J. (2018). Información científica en Argentina, España y México: fuentes, recursos multimedia y participación de los lectores en los diarios online. Estudios sobre el Mensaje Periodístico, 24(1), 397-412. https://doi.org/10.5209/ESMP.59957

Spina, G. D., \& Díaz, C. B. (2019). Jerarquización de las noticias sobre ciencia en los diarios digitales argentinos (2017-2018). Question, 1(61), 1-22. https://doi.org/10.24215/16696581e138

Summ, A., \& Volpers, A. M. (2016). What's science? Where's science? Science journalism in German print media. Public Understanding of Science, 25(7), 775-790. https://doi.org/10.1177/0963662515583419

Trench, B. (2007). How the Internet changed science journalism. In M. Bauer \& M. Massimiano (Eds.), Journalism, science and society: science communication between news and public relations (pp 133-142). Routledge. https://doi.org/10.4324/9780203942314

Williams, A., \& Clifford, S. (2009). Mapping the Field: Specialist Science News Journalism in the UK National Media. Cardiff University School of Journalism, Media and Cultural Studies.

Wimmer, R. D., \& Dominick, J. R. (1996). La investigación científica de los medios de comunicación de masas. Una introducción a sus métodos. Bosch Comunicación.

Wormer, H. (2008). Science journalism. In W. Donsbach (Ed.), The international encyclopedia of communication (pp. 4512-4514). England: Wiley-Blackwell.

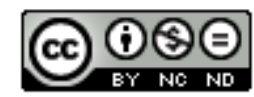

(C) Attribution-NonCommercial-NoDerivatives 4.0 International (CC BY-NC-ND 4.0) https://creativecommons.org/licenses/by-nc-nd/4.0/ 